\title{
BIOCHEMICAL AND HISTOLOGICAL CHANGES IN DEVELOPING RABBIT EYES*
}

\author{
BY
}

\section{B. A. BEMBRIDGE AND ANTOINETTE PIRIE}

From the Nuffield Laboratory of Ophthalmology, Oxford

AN attempt has been made to find the cells of origin and the time of origin during development of the vitreous hyaluronic acid. The adult vitreous body of those species so far examined seems to owe its characteristic jelly-like properties to the presence of small amounts of an insoluble collagen-like protein laid down in fibrous lamellae and a low concentration of hyaluronic acid. Earlier work with adult rabbits (Pirie, 1949) has shown that aggregated hyaluronic acid can be regenerated in the vitreous body after removal by hyaluronidase. It therefore seemed possible that, in the young, rapidly enlarging eye, histochemical tests might be able to show which cells produced hyaluronic acid and at what stage in development this took place. The time of production could then be checked by chemical analysis of the extracted vitreous body. Meyer (1947) has suggested that hyaluronic acid is secreted by the ciliary body.

We can say at once that we have no clear evidence on the origin of hyaluronic acid within the vitreous body; in pursuing this problem, however, we have collected information on changes in the chemical composition of the developing vitreous body in the young rabbit as well as histochemical evidence for the presence of a granular

- material in the retina at a certain stage of development which stains by the method for detecting polysaccharides (Hotchkiss, 1948). The evidence is briefly presented, as it may be of interest to, and save the the time of, others working on similar problems.

\section{Methods}

Material for Chemical Analysis.-Vitreous bodies dissected from rabbit eyes from birth onwards were used. The rabbits were killed by decapitation or nembutal injection and the eyes removed, washed, wiped free of all blood, and then opened by an equatorial cut. The vitreous body was shelled out and filtered through a glass filter. The filtrate was centrifuged to remove cells and pigment granules and the supernatant used for analysis. The vitreous filtrates from several eyes were pooled to get sufficient material.

Total Nitrogen was estimated by micro-Kjeldahl digestion followed by distillation and titration using the method of Markham (1942).

* Received for publication August 7, 1951. 
Hexosamine was estimated by the method of Elson and Morgan (1933) adapted for small quantities. Control estimations of glucosamine were always done in parallel, as well as a blank estimation of the vitreous filtrate to compensate for charring. Usually $0.5 \mathrm{ml}$. vitreous filtrate was taken for estimation. Colour was estimated in a Hilger Biochem absorptiometer or in a Beckman spectrophotometer.

Histological Investigation.-Eyes were fixed in Bouin's fluid and embedded in paraffin, and the sections were flattened on 70 per cent. alcohol for mounting on slides.

Subsequently these were prepared as follows:

(i) exactly according to the technique described by Hotchkiss (1948),

(ii) Best's carmine (Carleton and Leach, 1947),

(iii) Langhans' iodine (Carleton and Leach, 1947),

(iv) toluidine blue 1 per cent. (Lison, 1936; Sylvén, 1941),

(v) dialysed iron (Hale, 1946).

\section{RESULTS}

\section{Chemical Analyses of Developing Vitreous Humour}

Total Nitrogen of the adult rabbit vitreous humour lies at about $0.2 \mathrm{mg}$. N/ml., which corresponds to $1.25 \mathrm{mg}$. protein $/ \mathrm{ml}$. The vitreous of the newborn has about ten times this $\mathrm{N}$ content. Fig. 1 shows the changes in total $\mathrm{N}$ with age, and shows that the adult level is not reached until the rabbit is about 15 to 18 days old.

Hexosamine estimations taken as a measure of hyaluronic acid in a fluid containing possibly a small amount of hyaluronic acid and certainly a relatively large amount. of protein are liable to error. There is the error due to charring during hydrolysis which gives a yellow colour to the final

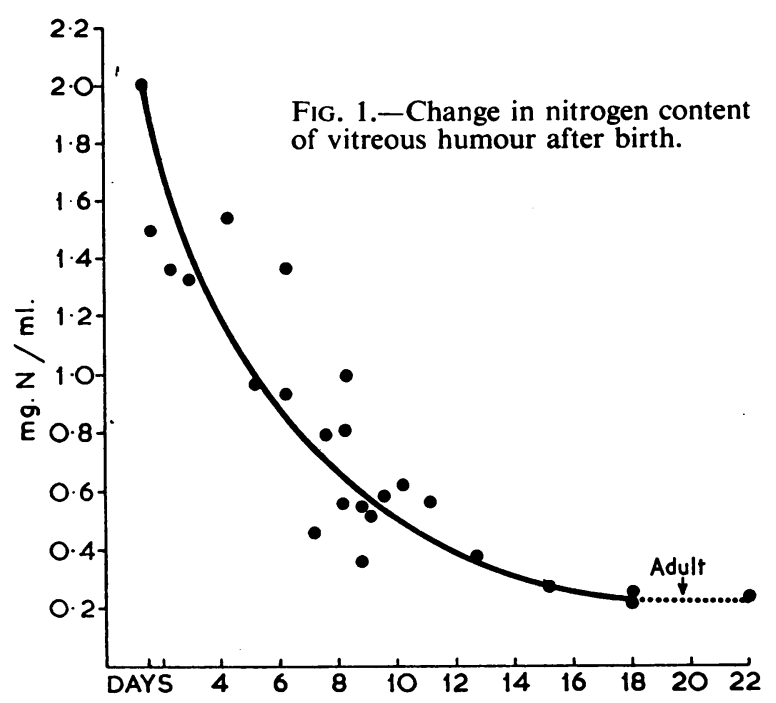
solution, which must be balanced during the colorimetric estimation; and there is the error due to possible interaction of amino-acids (liberated by hydrolysis) and glucose to give substances reacting like glucosamine in the estimation (Partridge and Gottschalk, 1950). The preformed glucosamine of the proteins, which Ogston and Stanier (1950) reckon as 1.5 per cent., has also to be allowed for. Small variations in hexosamine cannot, therefore, be taken as variations in hyaluronic acid content of the young vitreous. Bearing these reservations in mind, the hexosamine estimations show that, 
in all probability, there is no hyaluronic acid present in the vitreous body until about 10 days after birth. Fig. 12 shows the total hexosamine and the hyaluronic acid hexosamine taken as the total less the protein hexosamine.

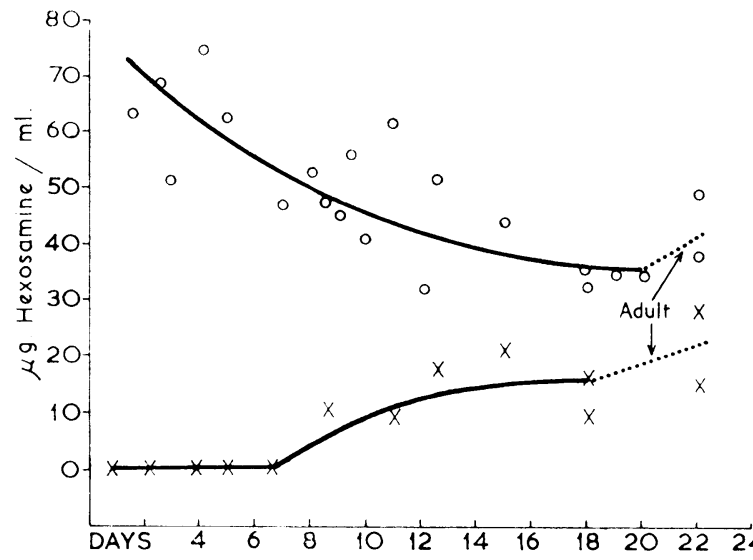

Fic. 2. - Change in total hexosamine $0-0$, and nonprotein hexosamine $x-\ldots x$, in vitreous humour after birth.

Histological Examination of the Developing Eye.-Chemical analysis showed that in all probability no hyaluronic acid was present in the vitreous body until about 10 days after birth. Hyaluronic acid is an acid polysaccharide and it reacts vigorously after oxidation with periodic acid in "spot tests" with Schiff's reagent. Any structure still containing it after fixation and preparation for histological sections should stain if the Hotchkiss technique is used. In all cases simultaneous controls were done by omitting the periodic acid oxidation from the procedure.

This histochemical method demonstrated the presence of "Hotchkiss positive " material in rabbit retina. This substance is absent both in the day-old rabbit retina and in the adult. It first appears at about the eighth day, in the form of extra-cellular, pink-staining granules, diffusely scattered through the retina. These granules cannot be found in normal rabbit retina after the third week. Their exact distribution varies, but they are mostly found in the inner retinal layers (Fig. 3).

Identification of the granules has not been carried further than placing them in the large group of substances which react with fuchsin sulphite (Schiff) after oxidation with periodic acid. Of these, possible substances are glycogen, mucins, hyaluronic acid, chondroitin sulphuric acid, heparin, and insoluble glycoproteins, such as those of the lens capsule and Descemet's membrane. The property of the retinal polysaccharide which has caused most difficulty in its identification has been its extreme solubility in aqueous solutions. Identification has been attempted by enzyme tests, and by determining if other staining techniques demonstrate the granules. Exposure of sections to distilled water or saline for five minutes at room temperature removed the staining material. It was therefore, impossible to determine whether hyaluronidase or salivary diastase digested the staining material, since this dissolved in the boiled enzyme or in saline controls. The granules are stable in 70 per cent. 
FIG. 3.-Granules in inner retinal layers stained by Hotchkiss method. 11-day-old rabbit. x 140 .

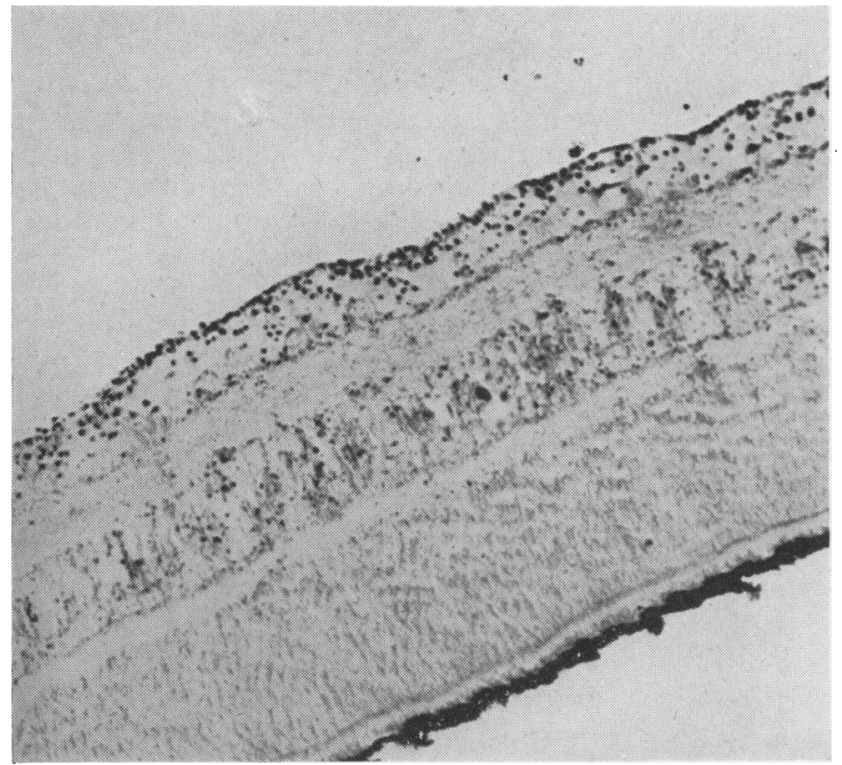

alcohol, but the enzymes are inactive in this solution. This extreme solubility also necessitated care in the fixation of the eyes, and we came to the conclusion that formalin fixation did not preserve the polysaccharide, but that Bouin's fluid did so. Sections of formol-fixed eyes showed no pink-staining granules, whereas Bouin-fixed eyes of the same age gave positive results. The difficulty in preserving the polysaccharide may also explain its varying distribution in the retinal layers, because as the fixative diffuses in, the polysaccharide may be disturbed by the diffusion currents, and its eventual extra-cellular appearance does not necessarily mean that it is extra-cellular in life.

This difficulty in obtaining adequate fixation is similar to that experienced in attempting to fix tissue containing glycogen. We found that Best's carmine stained the retinal granules; these were compared with granules found in liver stained with Best's carmine, in their behaviour towards salivary diastase and towards boiled enzyme. Here again there was difficulty in interpreting the controls.

The liver substance-presumably glycogen-which reacts with fuchsin sulphite after periodic acid was far less soluble than the retinal granules. Treatment of liver sections with boiled salivary diastase did not remove stainable material, whereas the active enzyme did so. The retina reacted quite irregularly, sometimes staining after the action of the boiled enzyme, but more often not staining.* Liver glycogen still gave positive results with the Hotchkiss method after exposure to boiled salivary diastase or distilled water for 15 to 20 minutes.

Attempts to demonstrate retinal glycogen granules with iodine have been

\footnotetext{
"In this technique, the slides were collodionized to prevent diffusion, which may explain why boiled salivary diastase occasionally did not remove the stainable material, whereas it always did so in non-
} collodionized slides using the Hotchkiss technique. 
unsuccessful, as was the use of Hale's dialysed iron to demonstrate hyaluronic acid.

The solubility of the polysaccharide may also account for the difficulty experienced in trying to demonstrate it metachromatically, using metachromasia as a demonstration of hyaluronic acid (Penney and Balfour, 1949). The basic metachromatic colouring agents require a watery solution for the metachromasia to appear, and the colour tends to disappear with dehydration. Thus this extemely soluble substance will tend to be washed out of a section by the very solution which is being used to demonstrate its presence. We were satisfied on one occasion only that there was metachromatic staining.

\section{DisCUSSION}

The vitreous body of the day-old rabbit is in its gross appearance the same as that of the adult, a glassy, clear jelly, yet the chemical analysis shows that it is in fact quite different. The hyaloid system does not degenerate in the rabbit until about 8 to 10 days after birth, and some of the extra protein of the vitreous is probably derived from this, but it is probable that there is a far greater concentration of protein in the young vitreous than in the adult, quite apart from the hyaloid system. Histologically it is easier to show a "structure" in the vitreous of the young or embryonic eye than in the adult eye, and it may be that precipitation of protein by the fixative gives the appearance of this " structure".

The rabbit eye does not open until the tenth day after birth, and Kinsey and others (1945) have shown that no secretion of ascorbic acid into the aqueous humour takes place until about this time. We have therefore three well-marked chemical changes going on in the eye of the rabbit in the first three weeks after birth: diminution of the protein of the vitreous body from the very high value at birth to the normal adult level of about $1.2 \mathrm{mg}$. protein $/ \mathrm{ml}$.; possibly, production of hyaluronic acid; secretion of ascorbic acid.

The soluble polysaccharide demonstrated in the retina of young rabbits may be glycogen. Glycogen is known to exist in varying states of solubility and is extremely difficult to preserve in very young and embryonic tissues (Lison, 1936). There have been many contradictory statements about the presence of glycogen in rabbit and frog retina and the part played by it in retinal metabolism. The most recent work appears to be a study of the frog retina by SchmitzMoorman (1927). He quotes Erhlich (1883) as demonstrating glycogen in frog retina; Brammertz (1915) in the frog and rabbit; and the contrary opinion of Best (1901-07). He found many variations in the distribution of glycogen in normal frog retina, and describes its presence in all layers, deciding that the constant and characteristic site was within the inner parts of the cones. His interpretation of this finding was that glycogen is used in the cone contractions which he' says occur in this animal. Nakashima (1926) 
found considerable glycogen in the pigment epithelial and rod layers of the rabbit retina after subcutaneous and intra-vitreal injection of dextrose. He suggested that the pigment epithelium plays a part in carbohydrate metabolism by taking up glucose from the choriocapillaris and converting it into glycogen.

Day (1950) did not find any polysaccharide granules in normal rabbit retina, but he was apparently not studying very young rabbits. In several eyes he found Hotchkiss positive material near the ciliary processes, but could draw no definite conclusions from the use of hyaluronidase, because he considered the enzyme he was using was not specific. MacDonald (1949), also using older rabbits, concluded that the site of hyaluronic acid formation is in the ciliary processes.

The rabbit eye at birth is-compared to a human eye at birth-a premature eye. The retina is not fully developed, the hyaloid system remains, and the lids are closed. If material were available it would be interesting to see whether similar chemical and histological changes take place in human eyes during this period of development.

\section{SUMmaRY}

(1) The protein of the rabbit vitreous body in the adult is approximately one-tenth of that in the newborn.

(2) There is probably no hyaluronic acid present in the newborn rabbit vitreous body.

(3) A soluble polysaccharide is present in rabbit retina from the eighth day to the end of the third week of life.

We wish to record our thanks to the National Council to Combat Blindness Inc., New York, for the Beckham spectrophotometer used in the later part of this work.

\section{REFERENCES}

BEST, F. (1905). Ber. ophthal. Ges. Heidelberg, 32, 315. (1906). Ibid., 33, 186. (1907). Arch. Augenheilk., 57, 173.

Brammertz, W. (1915). Arch. mikr. Anat., 86, 1.

Carleton, H. M., and Leach, E. H. (1947). "Histological Technique", p. 171. Oxford University Press, Oxford.

DAY, R. (1950). Amer. J. Ophthal., 33, 224.

Elson, L. A., and Morgan, W. T. J. (1933). Biochem. J., 27, 1824.

ERHLICH, B. (1883). Cited by F T. Frerichs: Z. klin. Med., 6, 33.

HAle, C. W. (1946). Nature, Lond., 157, 802.

Hotchkiss, R. D. (1948). Arch. Biochem., 16, 131.

KINSEY, V. E.. JACKSON, B., and TERRY, T. L. (1945). Arch. Ophthal., Chicago, 34, 415.

LisON, L. (1936). " Histochimie Animale", p. 226, 236. Gauthier-Villars, Paris.

MaCDonald, R. K. (1949). Amer. J. Ophthal., 32, June (ii), 96.

MARKHAM, R. (1942). Biochem, J., 37, 790.

MeYer, K. (1947). Physiol. Rev., 27, 335.

NAKASHima, C. (1926). v. Graefes Arch. Ophthal., 116, 403.

Ogston, A. G., and Stanier, J. (1950). Biochem. J., 46, 364.

Partridge, S. M., and GotTschalk, A. (1950). Nature, Lond., 165, 684.

Penney, J. R., and Balfour, B. M. (1949). J. Path. Bact., 61, 171.

PIRIE, A. (1949). British Journal of Ophthalmology, 33, 271.

Schmitz-Moorman, P. (1927). v. Graefes Arch. Ophthal., 118, 506.

Sylvén, B. (1941). Acta chir. scand., 86, suppl. $66,30$. 